\title{
Structural Changes of DPPC Bilayers Induced by Gemini Surfactant
}

\author{
W. KIDA, M. KOZAK \\ Departament of Macromolecular Physics, Faculty of Physics, A. Mickiewicz University, \\ Umultowska 85, 61-614 Poznań, Poland
}

\begin{abstract}
The phospholipid-gemini surfactant systems are promising agents for construction of lipoplexes for DNA delivery systems in gene therapy. In this work the influence of a gemini surfactant - 1,1'-(1,6-hexan)bis3octyloxymethylimidazolium di-chloride) (IMI_Cl_C6_C8) on the structure and phase behaviour of aqueous suspensions of the fully hydrated phospholipid - 1,2-dipalmitoyl-sn-glycero-3-phosphocholine (DPPC) were characterised using small angle scattering of synchrotron radiation (SAXS), differential scanning calorimetry (DSC) and Fourier transform infrared spectroscopy (FTIR). The surfactant studied caused a destabilisation of the lamellar phase typical of DPPC. Addition of the surfactant also shifted to lower temperatures and extended the range of the main phase transition
\end{abstract}

PACS: 87.15.Zg, 61.05.cf, 87.64.km, 87.16.dt

\section{Introduction}

Recently, increasing demand for phospholipid/surfactant mixed systems has been observed. They are used in numerous areas of research and industrial applications. Of particular interest are their applications in cosmetics [1], pharmaceuticals [2-4] or structural biology $[5,6]$. Most of these systems are based on classical monomeric surfactants.

In the early 1980s a new group of surfactants made up of two amphiphilic moieties connected by a rigid or flexible spacer group (at the level of the head groups or very close to the head groups) were synthesised [7]. This group of surfactants is commonly known as gemini surfactants [8], but they also have been referred to as dimeric surfactants [9], bis-quatenary ammonium surfactants [10], dicationic detergents [11] as well as siamese surfactants (by analogy to Siamese twins) [12]. This new group of surfactants is characterised by three major benefits in comparison to monomeric surfactants $[7,13]$. The critical micellization concentration (cmc) is much lower than $\mathrm{cmc}$ characterising the homological monomeric surfactants (usually one to two orders of magnitude). The dimeric surfactants are much more effective in decreasing the surface tension of water than the corresponding monomeric surfactants [13, 14]. Moreover, the aqueous solutions of the gemini surfactants with short spacers at relatively low surfactant concentrations show much higher viscosity than the solutions of the corresponding monomeric surfactants [13, 14].

Symmetric dicationic $(\mathrm{m}-\mathrm{s}-\mathrm{m})$ surfactants with a short spacer group exhibit also significantly higher antimicrobial activity (even 100 times higher) than the monomeric surfactants commonly used as germicides (dodecylbenzyldimethylammonium bromide or 2-ethoxycarbonylpentadecyltri-methylammonium bromide) [15, 16]. This group of surfactants has also promising applications in preparation of cosmetics, emulsifiers, personal care products etc. Therefore, understanding the influence of the gemini surfactants on the structure of typical structural phases of phospholipids is very important for applications (cosmetics, drug delivery systems, vaccines) [17-19] as well as for basic research (for example structural biology) [20].

Dipalmitoylphosphatidylcholine (DPPC) is a phospholipid and also a major component of cell membranes as well as is often used as a model system in the studies of behaviour of lipid bilayers. Dicationic (gemini) surfactants can strongly interact with phospholipids and induce changes in the structure of phospholipid phases.

The aim of this work was to analyse the influence of a gemini surfactant, 1,1'-(1,6-hexan)bis 3-octyloxymethylimidazolium di-chloride, (IMI_Cl_C6_C8) on the structure and phase behaviour of aqueous suspensions of the fully hydrated 1,2-dipalmitoyl-sn-glycero-3phosphocholine (DPPC). In this work the interactions of a gemini surfactant with DPPC were characterised using small angle X-ray scattering (SAXS), differential scanning calorimetry (DSC) and Fourier transform infrared spectroscopy (FTIR).

\section{Experimental}

\subsection{Sample preparation}

The dicationic surfactant IMI Cl C6 $\quad$ C8 $\quad$ (see Fig. 1a) was prepared as described in [21] The phospholipid sample - 1,2-dipalmitoyl-sn-glycero-3phosphocholine (DPPC) was purchased from Avanti Polar Lipids (USA) (Fig. 1b). The reference samples, which were a homogeneous $10 \%(\mathrm{w} / \mathrm{w})$ solution of DPPC (in $20 \mathrm{mM} \mathrm{K} \mathrm{HPO}_{4} / \mathrm{D}_{2} \mathrm{O} \mathrm{pH} 6.5$ ) and also phospholipid/surfactant mixtures (with lipid/surfactant molar 
ratio $200: 1,100: 1,20: 1,10: 1$ and 5:1 1,1'-(1,6-hexan)bis3octyloxymethylimidazolium di-chloride were prepared by the 10 cycles of sonication at $320 \mathrm{~K}$ for $30 \mathrm{~min}$ and cooling at $278 \mathrm{~K}[22]$.

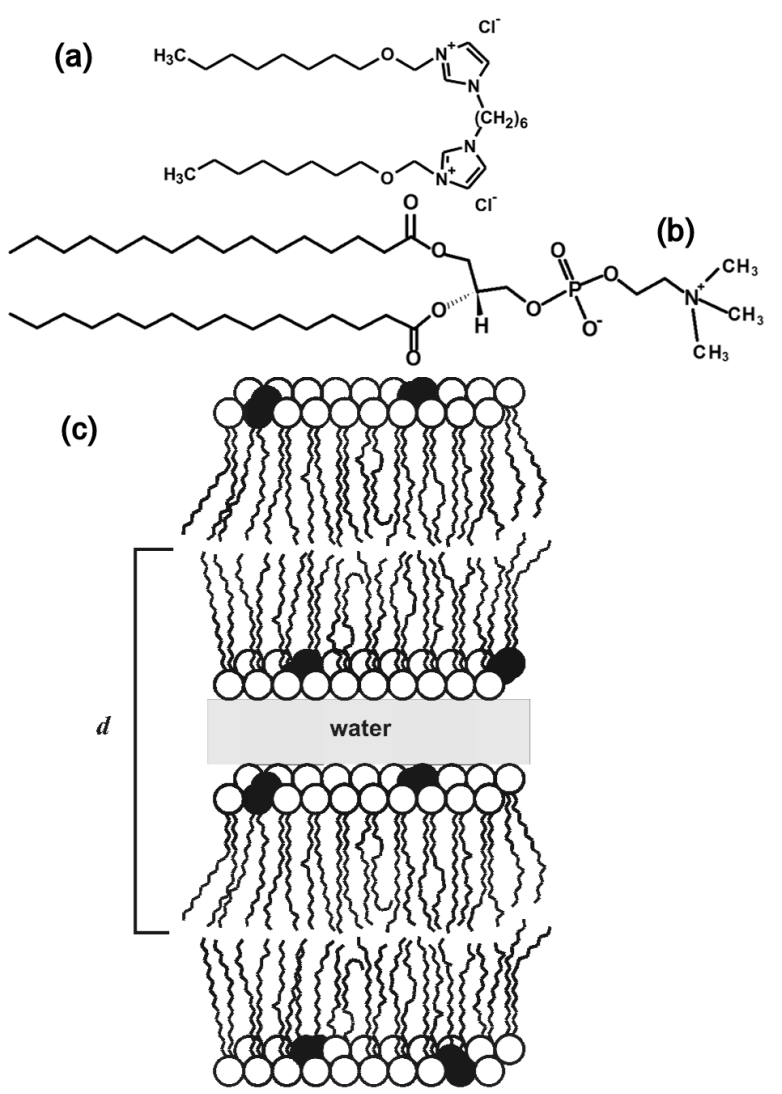

Fig. 1. Chemical structures of the gemini surfactant used - 1,1'-(1,6-hexan)bis3octyloxymethylimidazolium dichloride (a) and 1,2dipalmitoyl-sn-glycero-3-phosphocholine (DPPC) (b) and schematic representation of liquid-crystalline phase $\left(\mathrm{L}_{\alpha}\right)$ formed by hydrated DPPC (white) and gemini surfactant (black) (c).

\subsection{SAXS studies}

The X-ray scattering data were collected at the MAXII storage ring of the MAX-Lab (Lund, Sweden) on the Beam Line I911-4 [23] using the synchrotron radiation $(\lambda=0.091 \mathrm{~nm})$ and the MarCCD $165 \mathrm{~mm}$ detector. The phospholipid-surfactant samples $\left(0.1 \mathrm{~cm}^{3}\right)$ were placed in a thermostated capillary sample holder. The data were collected at temperatures ranging from 278 to $318 \mathrm{~K}$. The scattering pattern for the buffer solution (background scattering) was collected before and after data collection for lipid samples. The scattering vector (s-axis) was calibrated using silver behenate [24]. The sample-to-detector distance was $1.9 \mathrm{~m}$, which leads to the scattering vector range $0.05<\mathrm{s}<4.81 \mathrm{~nm}^{-1}$ (where $s=4 \pi \sin \theta / \lambda, 2 \theta$ is the scattering angle and $\lambda$ is the synchrotron radiation wavelength). Only SAXS data for reference DPPC system were collected within narrower s range $(0.05<\mathrm{s}$ $<3.4 \mathrm{~nm}^{-1}$ ).

For all data sets the standard procedures of normalization, correction for incident beam intensity, correction for detector response and integration of images were applied. These procedures were performed by the use of SAXS data reduction software Bli711 [23]. The scattering data of the buffer was subtracted from SAXS data of the sample using the program package PRIMUS [25] and the positions of the diffraction picks on the SAXS curves were fitted using the program PEAK [25].

\subsection{FTIR measurements}

The infrared absorption spectra were collected using CONFOCHECK system. This system is based on FTIR Bruker spectrometer TENSOR 27 equipped in the AquaSpec micro transmission cell. The samples of about $10 \mu \mathrm{l}$ were placed in a flow-through cuvette with a path length of approximately $7 \mu \mathrm{m}$. and incubated for 90 seconds at a given temperature before starting the experiment. The FTIR data were collected in the temperature range from 277 to $329 \mathrm{~K}$ using HAAKE DL30 thermostat. For each spectrum 128 scans in the spectral range $4000-950 \mathrm{~cm}^{-1}$ were collected with the resolution of 2 $\mathrm{cm}^{-1}$. The original FTIR spectra were smoothed using the Savitzky-Golay method. The smoothing procedure was taken over 5 data points.

\subsection{Differential scanning calorimetry (DSC)}

The calorimetric measurements were carried out using Netzsch Phoenix DSC-204 system equipped with an $\mathrm{m}$-sensor. The liquid samples of average mass $25 \mathrm{mg}$ $\pm 5 \%$ were hermetically enclosed in aluminium crucibles. The samples were initially isothermally incubated for 10 minutes at $T=273 \mathrm{~K}$. For each sample two successive dynamic scans from $273 \mathrm{~K}$ to $353 \mathrm{~K}$ were collected in the helium atmosphere with the scan rate of $1 \mathrm{~K} / \mathrm{min}$. The DSC data were analysed by a computer program TA (Netzsch). The phase transition enthalpy was calculated using the linear or tangent-sigmoidal base line. For complex curves the peak-separation procedure using the Fraser-Suzuki profile was applied.

\section{Results and discussion}

Fully hydrated DPPC was found to undergo three phase transitions typical of phospatidyl choline derivateves: subtransition (crystalline gel to lamellar gel, $\mathrm{L}_{c^{\prime}} \rightarrow \mathrm{L}_{\beta^{\prime}}$, at $291 \mathrm{~K}$ ), pretransition (lamellar gel to rippled gel, $\mathrm{L}_{\beta^{\prime}} \rightarrow \mathrm{P}_{\beta^{\prime}}$, at $307 \mathrm{~K}$ ) and main transition (lamellar rippled gel to lamellar liquid-crystalline, $\mathrm{P}_{\beta^{\prime}} \rightarrow \mathrm{L}_{\alpha}$ at $314.5 \mathrm{~K}$ ) [26].

The scattering data collected for the reference system of pure $10 \%$ DPPC are presented in Fig. 2. On the basis of SAXS data the $d$-spacing values were calculated and are summarized in Fig. 3. The structural phases characteristic of DPPC were observed. In this system 
the crystalline gel phase was observed with a bilayer $d$ spacing: $d_{001}=6.3 \mathrm{~nm}, d_{002}=3.15 \mathrm{~nm}, d_{003}=2.1 \mathrm{~nm}$ (at $278 \mathrm{~K}$ ). Above the temperature of $308 \mathrm{~K}$ the bilayer thickness increased rapidly. At $313 \mathrm{~K}, \mathrm{~L}_{\alpha}$ phase was characterized by $d$-spacing 7.11 and $3.67 \mathrm{~nm}$. These values are in good agreement with the previously reported ones [26-28]. The SAXS data collected for different surfactant concentrations in the systems are presented in Figs. 48 The increasing surfactant concentration in the mixed lipid/surfactant system resulted in gradual weakening followed by total disappearance of the strong diffraction maxima (see $d$-spacing changes in Fig. 3). Such an effect was observed also for other monomeric and gemini surfactants $[21,29,30]$.

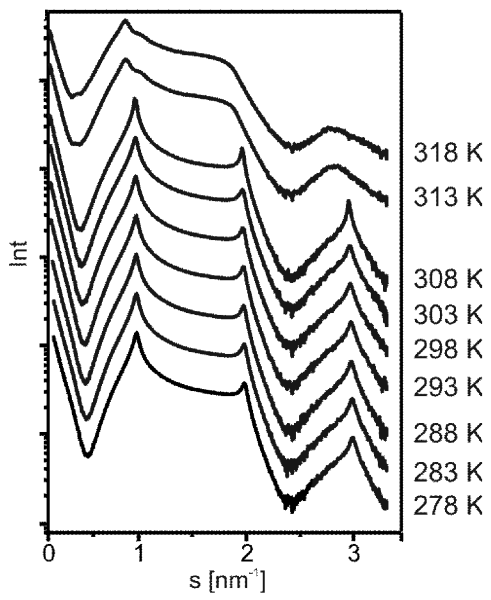

Fig. 2. SAXS data for the references system - 10\% DPPC in $\mathrm{D}_{2} \mathrm{O}$ (temperature range $278-318 \mathrm{~K}$ ). The data were shifted for clarity.

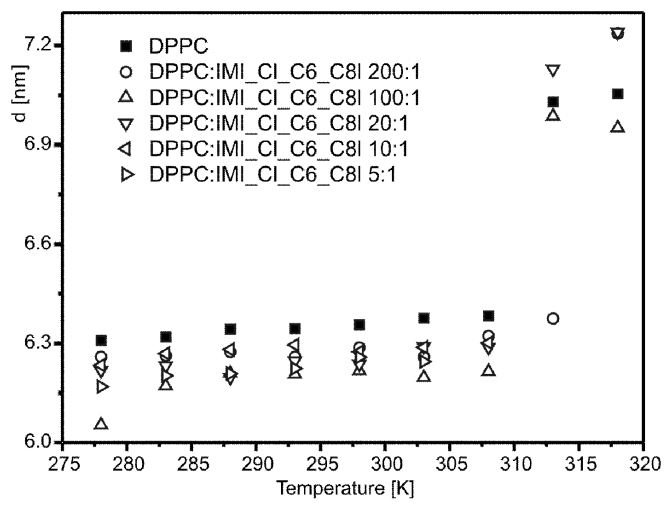

Fig. 3. The values of $d_{001}$ versus temperature for all systems studied.

For the lowest concentrations of the surfactant (200:1 and 100:1) the bilayer spacing after the main transition increased up to $7.2 \mathrm{~nm}$ (see schematic representation of mixed $\mathrm{L}_{\alpha}$ phase in Fig. 1c). Further addition of the surfactant to the system resulted in the disappearance of the diffraction peak (Figs. 6, 7). In the systems with the

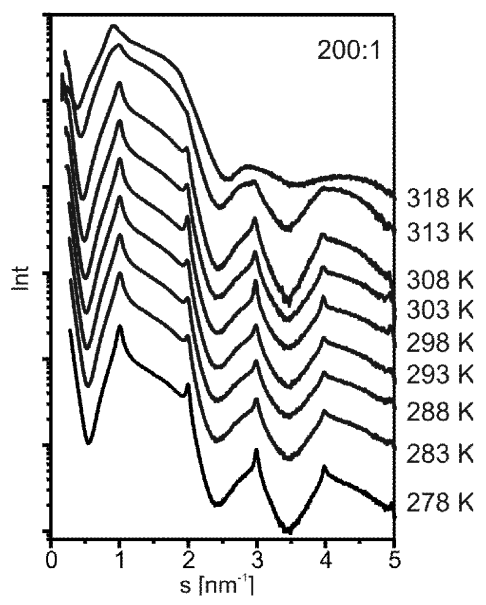

Fig. 4. SAXS data for the system - DPPC/IMI C6 $\mathrm{C} 8 \mathrm{Cl} 200: 1$ (temperature range $278-318 \mathrm{~K}$ ). The data were shifted for clarity.

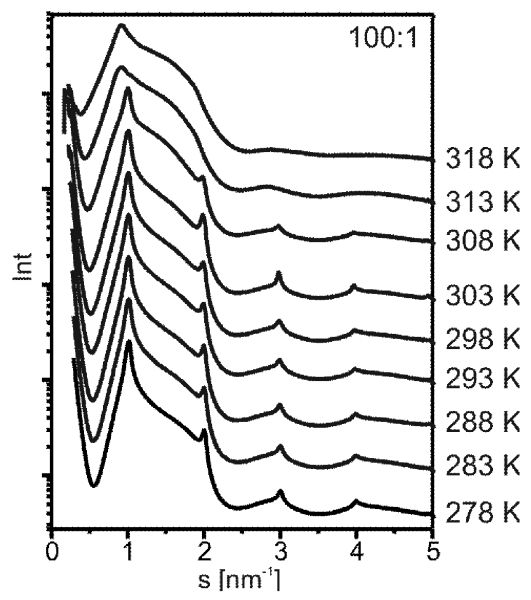

Fig. 5. SAXS data for the system - DPPC/IMI C6_C8Cl 100:1 (temperature range 278-318 K). The data were shifted for clarity.

highest concentrations of the surfactant (10:1 and 5:1) the diffraction peaks almost completely disappear and very broad maxima are observed in the s-vector range from 0.5 to $2.5 \mathrm{~nm}^{-1}$. Very wide maxima may suggest the presence of phases without bilayers stacking (probably formation of the bicellar phase) [31-33]. Unfortunately the system was not monodisperse and the calculation of the pair distance distribution function $p(r)$ and $\mathrm{D}_{\max }$ characterizing bicelles was impossible.

The reference sample - fully hydrated DPPC and DPPC/surfactant systems were also analyzed by FTIR. The analysis focused in particular on the band in the range from 2800 to $3000 \mathrm{~cm}^{-1}$. This part of the infrared spectrum is assigned to the vibrations of the polymethylene chain (for example see Fig. 9) and provides detail information on the conformation of alkyl chains of DPPC. Gauche conformers of polymethylene chains $\left(\left(\mathrm{CH}_{2}\right)_{n}\right)$ of 


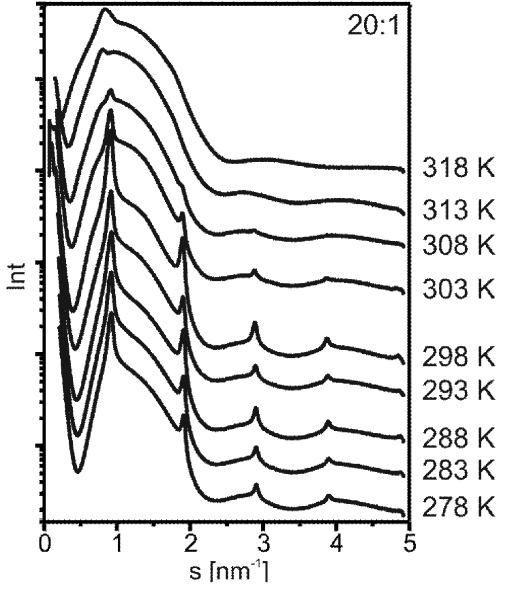

Fig. 6. SAXS data for the system - DPPC/IMI C6 $\mathrm{C} 8 \mathrm{Cl}$ 20:1 (temperature range $278-318 \mathrm{~K}$ ). The data were shifted for clarity.

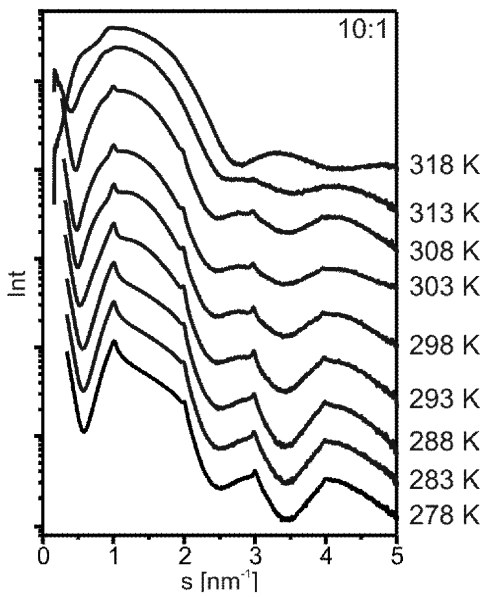

Fig. 7. SAXS data for the system - DPPC/IMI C6 $\mathrm{C} 8 \mathrm{Cl}$ 10:1 (temperature range $278-318 \mathrm{~K}$ ). The data were shifted for clarity.

phospholipid absorb infrared radiation at higher frequencies than those containing all-trans conformers [34].

The temperature-dependent changes in the wave numbers characterizing the $\mathrm{CH}_{2}$ symmetric and anti-symmetric stretching vibrations for DPPC and DPPC/surfactant systems are presented in Figs. 10 and 11. The temperature of the main phase transition, which is connected with full trans/gauche reorientation was estimated on the basis of $\mathrm{CH}_{2}$ symmetric and anti-symmetric stretching vibration in pure DPPC and was exactly the same $(315 \mathrm{~K})$. The increasing surfactant concentration in the systems studied caused a decrease in the temperature of the main transition. It was caused by surfactant building up in the DPPC bilayers which induced some local structural disorder and made space for trans gauche izomerization. Addition of the surfactant also extended the range of the main phase transition and

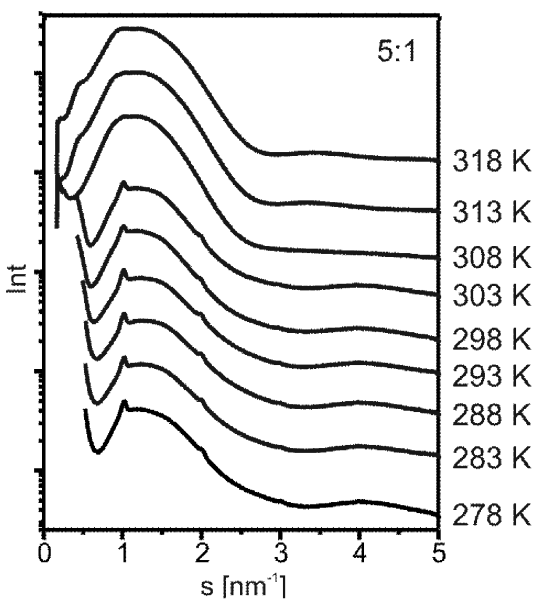

Fig. 8. SAXS data for the system - DPPC/IMI C6 $\mathrm{C} 8 \mathrm{Cl}$ 5:1 (temperature range $278-318 \mathrm{~K}$ ). The data were shifted for clarity.

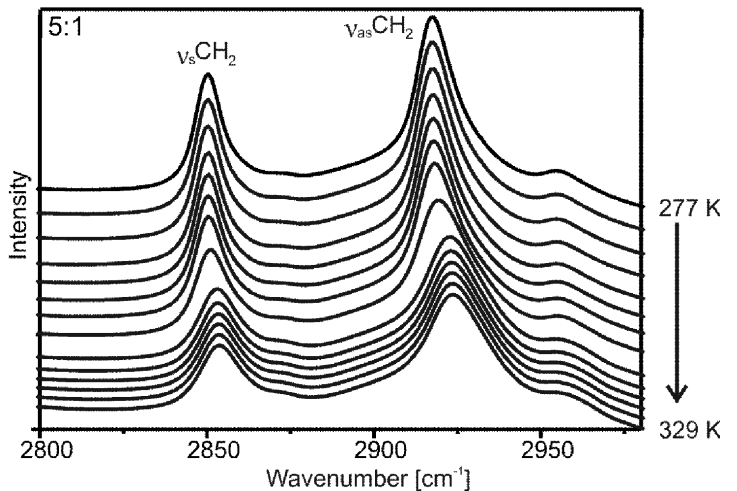

Fig. 9. The example of FTIR absorption spectra for DPPC/IMI_Cl_C6_C8 5:1 in the region analyzed.

this phenomena was observed for other systems lipids mixed with different compounds (vitamins, steroids or surfactants) as well [35-37].

The SAXS and FTIR studies were supported by DSC results. It is well documented that phase transitions observed in DPPC systems can be visualized by differential scanning calorimetry [38, 39]. DSC thermograms obtained for the reference DPPC and DPPC/surfactant systems are shown in Fig. 12. The parameters characterizing phase transitions in the systems studied are summarized in Table. In the reference DPPC system, the main transition was characterized by $T_{\text {onset }}=314.9 \mathrm{~K}$ with enthalpy of $\Delta H=8.6 \mathrm{kcal} / \mathrm{mol}$ The addition of the surfactant decreased the temperature characterizing the main transition (up to $T_{\text {onset }}=305.5 \mathrm{~K}$ for DPPC/surfactant system 5:1) and changed the transition enthalpy (up to $4.6 \mathrm{kcal} / \mathrm{mol}$ for DPPC $/$ surfactant system 10:1). Such phenomena were also observed for other lipid/surfactant systems [40, 41]. 
TABLE

The thermodynamic parameters characterising the reference DPPC and DPPC/gemini surfactant systems

\begin{tabular}{l|c|c|c}
\hline \hline $\begin{array}{l}\text { DPPC: } \\
\text { IMI_Cl_C6_C8 }\end{array}$ & $\begin{array}{c}\Delta H \\
{[\mathrm{kcal} / \mathrm{mol}]}\end{array}$ & $\begin{array}{c}T_{\text {peak }} \\
{[\mathrm{K}]}\end{array}$ & $\begin{array}{c}T_{\text {onset }} \\
{[\mathrm{K}]}\end{array}$ \\
\hline Reference system & 8.6 & 314.9 & 314.6 \\
$200: 1$ & 8.8 & 314.1 & 313.5 \\
$100: 1$ & 5.9 & 314.3 & 313.5 \\
$20: 1$ & 6.1 & 312.1 & 310.6 \\
$10: 1$ & 4.6 & 310.1 & 306.4 \\
$5: 1$ & 5.8 & 307.4 & 305.5
\end{tabular}

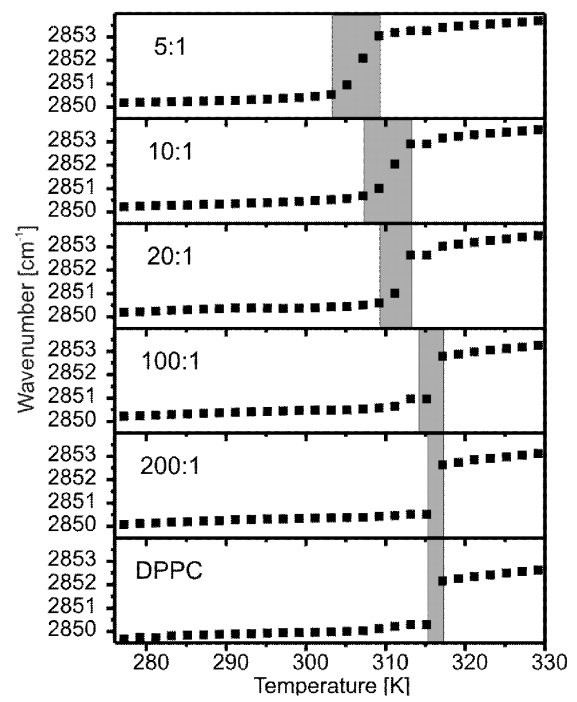

Fig. 10. Temperature dependence of $\mathrm{CH}_{2}$ symmetric stretching.

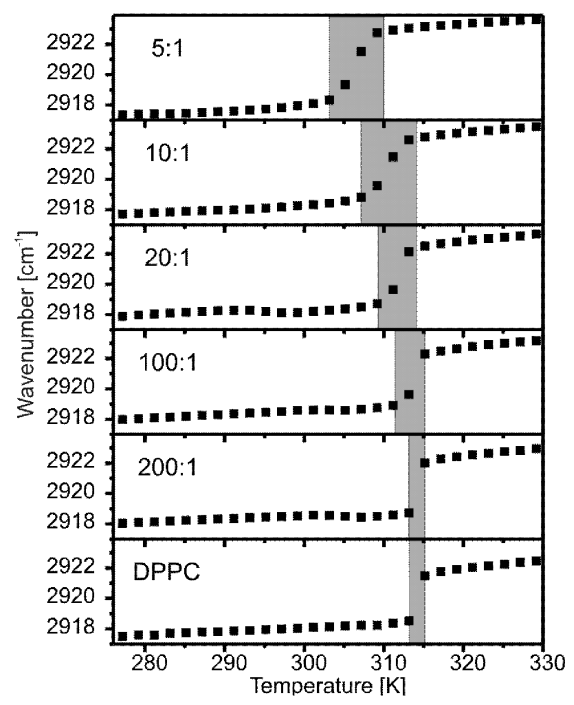

Fig. 11. Temperature dependence of $\mathrm{CH}_{2}$ antisymmetric stretching.

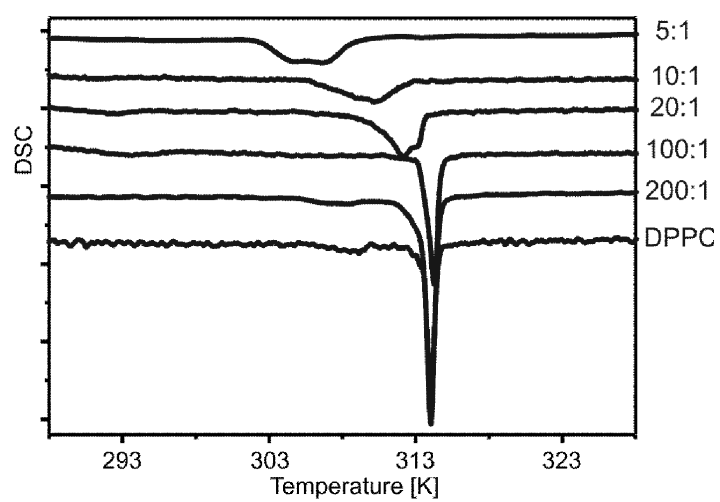

Fig. 12. DSC thermograms (heating) of DPPC bilayers in the presence of increasing concentrations of gemini surfactant.

\section{Conclusions}

The surfactant studied caused a destabilisation of the lamellar phase typical of DPPC. For the highest surfactant concentrations the scattering patterns observed for DPPC/gemini surfactant systems are very similar to the SAXS patterns reported for the classical bicellar DPPC/DHPC systems.

This effect is also observed by DSC as a broadening of the temperature range of the main phase transition and a decrease in the phase transition enthalpy. FTIR results show decreasing temperature of the phase transition and changes in the wavenumber characteristics of the vibrations of $\nu_{a s} \mathrm{CH}_{2}$ and $\nu_{s} \mathrm{CH}_{2}$ groups.

\section{Acknowledgments}

The research was supported by research grant the Ministry of Science and Higher Education (Grant No.N N204 135738). The research (the data collection at Beam Line I911) leading to these results has also received funding from the European Community's Seventh Framework Programme (FP7/2007-2013) under grant agreement no. 226716 .

\section{References}

[1] H. Heerklotz, Q. Rev. Biophys. 41, 205 (2008).

[2] S. Jain, P. Jain, R.B. Umamaheshwari, N.K. Jain, Drug Dev. Ind. Pharm. 29, 1013 (2003).

[3] M.J. Lawrence, G.D. Rees, Adv. Drug Deliv. Rev. 45, 89 (2000).

[4] M. Kreilgaard, Adv. Drug Deliv. Rev. 54, S77 (2002).

[5] D.E. Warschawski, A.A. Arnold, M. Beaugrand , A. Gravel, É. Chartrand, I. Marcotte, Biochim. Biophys. Acta-Biomembr. 1808, 1957 (2011).

[6] M. Caffrey, Annu. Rev. Biophys. 38, 29 (2009).

[7] R. Zana, in: Novel Surfactants: Preparation, Applications and Biodegradability, M. Dekker Inc, New York (1998). 
[8] F.M. Menger, C.A. Littau, J. Am. Chem. Soc. 113 , 1451 (1991).

[9] R. Zana, M. Benrraou, R. Rueff, Langmuir 7, 1072 (1991).

[10] H.C. Parreira, E.R. Lukenbach, M.K. Lindemann, J. Am. Oil Chem. Soc. 56, 1015 (1979).

[11] C.A. Bunton, L. Robinson, J. Schaak, M.F. Stam, J. Org. Chem. 36, 2346 (1971).

[12] M. Frindi, B. Michels, H. L'evy, R. Zana, , Langmuir 10, 1140 (1994).

[13] R. Zana, Gemini Surfactants: Synthesis, Interfacial and Solution-Phase Behavior, and Applications, CRC Press (2003).

[14] R. Zana, Adv. Colloid Interface Sci. 97, 205 (2002).

[15] M. Pavlikova, I. Lacko, F. Devinsky, D. Mlynarcik, Collect. Czech. Chem. Commun. 60, 1213 (1995).

[16] M. Dubnickova, M. Pisarcik, I. Lacko, F. Devinsky, Cell. Mol. Biol. Lett. 2, 215 (1997).

[17] S. Svenson, Curr. Opin. Colloid Interface Sci. 9, 201 (2004).

[18] C. Bombelli, L. Giansanti, P. Luciani, G. Mancini, Curr. Med. Chem. 16, 171 (2009).

[19] S.K. Hait, S.P. Moulik, Curr. Sci. 82, 1101 (2002).

[20] D. Blunk, P. Bierganns, N. Bongartz, R. Tessendorf, C. Stubenrauch, New J. Chem. 30, 1705 (2006).

[21] Z. Pietralik, M. Taube, A. Skrzypczak, M. Kozak, Acta Phys. Polon. A 117, 311 (2010).

[22] Á. Csiszár, E. Klumpp, A. Bota, K. Szegedi, Chem. Phys. Lipids 126, 155 (2003).

[23] M. Knaapila, C. Svensson, J. Barauskas, M. Zackrisson, S.S. Nielsen, N. Nørgaard Toft, B. Vestergaard, L. Arleth, Y. Cerenius, J. Synch. Rad. 16, 498 (2009).

[24] T.C. Huang, H. Toraya, T.N. Blanton, Y. Wu, J. Appl. Cryst. 26, 180 (1993).

[25] P.V. Konarev, V.V. Volkov, A.V. Sokolova, M.H.J. Koch, D.I. Svergun, J. Appl. Cryst. 36, 1277 (2003).

[26] M. Rappolt, G. Pabst, G. Rapp, M. Kriechbaum, H. Amenitsch, Ch. Krenn, S. Bernstorff, P. Laggner, Eur. Biophys. J. 29, 125 (2000).
[27] T. Mavromoustakos, E. Theodoropoulou, D. Papahatjis, T. Kourouli, ve-Ping Yang, M. Trumbore, A. Makriyannis, Biochim. Biophys. Acta-Biomembr. 1281, 235 (1996).

[28] B. Carion-Taravella, S. Lesieur, J. Chopineau, P. Lesieur, M. Ollivon. Langmuir 18, 325 (2002).

[29] E. Soussan, M. Blanzat, I. Rico-Lattes, A. Brun, C.V. Teixeira, G. Brezesinski, F. Al-Ali, A. Banu, M. Tanaka, Colloid Surf. A-Physicochem. Eng. Asp. 303, 55 (2007).

[30] M. Kozak, L. Domka, S. Jurga, J. Therm. Anal. Calorim. 88, 395 (2007).

[31] J. Bolze, T. Fujisawa, T. Nagao, K. Norisada, N. Saito, A. Naito, Chem. Phys. Lett. 329, 215 (2000).

[32] P. Laggner, J.D. Gotto, J.D. Morrisett, Biochemistry 18, 164 (1979).

[33] M. Kozak, M. Kempka, K. Szpotkowski, S. Jurga, J. Non-Cryst. Solids 353, 4246 (2007).

[34] S.W. Huffman, S. Schlucker, I.W. Levin, Chem. Phys Lipids 130, 167 (2004).

[35] H. Boyar, F. Severcan J. Mol. Struct. 408/409, 269 (1997).

[36] F. Severcan, I. Sahin, N. Kazanci, Biochim. Biophys Acta-Biomembr. 1668, 215 (2005).

[37] M. Kozak, K. Szpotkowski, A. Kozak, R. Zieliński, D. Wieczorek, M.J. Gajda, L. Domka, Radiat. Phys. Chem. 78, S129 (2009).

[38] X. Zhai, M. Bartel, G. Brezesinski, B. Rattay, H. Mohwald, J. Li. Chem. Phys. Lipids 133, 79 (2005).

[39] R. Koynova, M. Caffrey, Biochim. Biophys. Acta 1376, 91 (1998).

[40] C. Matsingou, C. Demetzos, Chem. Phys. Lipids 145, 45 (2007).

[41] M. Kozak, K. Szpotkowski, A. Kozak, R. Zielinski, D. Wieczorek, M.J. Gajda, Radiat. Phys. Chem. 78, S112 (2009). 